\title{
Antipsychotic Drug Prescription in Pediatric Intensive Care Units: A 10-Year U.S. Retrospective Database Study
}

\author{
Kate Madden ${ }^{10}$ Michael Wolf ${ }^{2}$ Robert C. Tasker ${ }^{1}$ Janet Figueroa ${ }^{2}$ Courtney McCracken ${ }^{2}$ \\ Matt Hall ${ }^{3}$ Pradip Kamat ${ }^{2,4}$
}

${ }^{1}$ Department of Anesthesiology, Critical Care, and Pain Medicine, Boston Children's Hospital, Boston, Massachusetts, United States

2 Department of Pediatrics, Emory University School of Medicine, Atlanta, Georgia, United States

${ }^{3}$ Children's Hospital Association, Lenexa, Kansas, United States

${ }^{4}$ Division of Critical Care, Children's Healthcare of Atlanta at Egleston, Atlanta, Georgia, United States

\begin{abstract}
Address for correspondence Kate Madden, MD, Department of Anesthesiology, Critical Care and Pain Medicine, Boston Children's Hospital, 300 Longwood Avenue, Boston, MA 02115, United States (e-mail: kate.madden@childrens.harvard.edu).
\end{abstract}

J Pediatr Intensive Care

\begin{abstract}
Keywords

- antipsychotic

- delirium

- pediatric critical care

Delirium recognition during pediatric critical illness may result in the prescription of antipsychotic medication. These medications have unclear efficacy and safety. We sought to describe antipsychotic medication use in pediatric intensive care units (PICUs) contributing to a U.S. national database. This study is an analysis of the Pediatric Health Information System Database between 2008 and 2018, including children admitted to a PICU aged 0 to 18 years, without prior psychiatric diagnoses. Antipsychotics were given in 16,465 (2.3\%) of 706,635 PICU admissions at 30 hospitals. Risperidone (39.6\%), quetiapine (22.1\%), and haloperidol (20.8\%) were the most commonly used medications. Median duration of prescription was 4 days (interquartile range: 2-11 days) for atypical antipsychotics, and haloperidol was used a median of 1 day (1-3 days). Trend analysis showed quetiapine use increased over the study period, whereas use of haloperidol and chlorpromazine (typical antipsychotics) decreased $(p<0.001)$. Compared with no antipsychotic administration, use of antipsychotics was associated with comorbidities ( 81 vs. $65 \%$ ), mechanical ventilation (57 vs. $36 \%$ ), longer PICU stay (6 vs. 3 days), and higher mortality (5.7 vs. $2.8 \%$ ) in univariate analyses. In the multivariable model including demographic and clinical factors, antipsychotic prescription was associated with mortality (odds ratio $[O R]=1.09$, 95\% confidence interval $[\mathrm{Cl}]: 1.02-1.18)$. Use of atypical antipsychotics increased over the 10-year period, possibly reflecting increased comfort with their use in pediatric patients. Antipsychotics were more common in patients with comorbidities, mechanical ventilation, and longer PICU stay, and associated with higher mortality in an adjusted model which warrants further study.
\end{abstract}

received

July 10, 2021

accepted after revision

August 31, 2021
DOI https://doi.org/

$10.1055 / \mathrm{s}-0041-1736523$.

ISSN 2146-4618.

\footnotetext{
(C) 2021. The Author(s).

This is an open access article published by Thieme under the terms of the Creative Commons Attribution-NonDerivative-NonCommercial-License, permitting copying and reproduction so long as the original work is given appropriate credit. Contents may not be used for commercial purposes, or adapted, remixed, transformed or built upon. (https://creativecommons.org/ licenses/by-nc-nd/4.0/) Georg Thieme Verlag KG, Rüdigerstraße 14, 70469 Stuttgart, Germany
} 


\section{Introduction}

Awareness of the prevalence of delirium among children admitted to pediatric intensive care units (PICUs) and pediatric cardiac intensive care units (PCICUs) has been increasing over the past decade. ${ }^{1-7}$ This awareness and the availability of pediatric delirium screening tools have led to the development of delirium management pathways which include both nonpharmacologic and pharmacologic approaches. ${ }^{8-10}$ When nonpharmacologic measures are insufficient, antipsychotic agents are often suggested. As no antipsychotic agent is approved by the U.S. Food and Drug Administration (FDA) for treatment of delirium in children, antipsychotics are administered off-label in PICUs and PCICUs. Several studies have reported single-center experience with antipsychotics for PICU delirium with varying report of adverse events. ${ }^{11-14}$ One study reported adverse events in $9.6 \%$ of patients treated with haloperidol, while another study reported a $5 \%$ incidence in children prescribed quetiapine, largely QT prolongation. ${ }^{15,16}$ No studies have demonstrated efficacy of antipsychotic agents in pediatric delirium; a recent single-center cohort of 27 PICU patients demonstrated worsened delirium scores with haloperidol. ${ }^{17}$ Results of several large adult ICU studies have conflicting results regarding benefits to ICU outcomes. ${ }^{18-20}$

Increasing awareness of PICU delirium and more systematic pediatric delirium screening is starting to expand to include other hospitalized pediatric patients. In the context of the lack of published efficacy for delirium, we sought to evaluate recent use of antipsychotics in PICUs and PCICUs, and how it may have changed over the past decade.

\section{Materials and Methods}

\section{Study Design and Setting}

This retrospective database study used the Pediatric Health Information System (PHIS) database which contains administrative and billing data from 49 tertiary care children's hospitals located in 27 states and the District of Columbia. The PHIS database is maintained by the Children's Hospital Association in Lenexa of Kansas which along with participating hospitals jointly ensures data integrity and quality. The PHIS hospitals provide discharge data, including patient demographics, diagnoses, and procedure codes for all inpatient, observation, emergency department, and ambulatory surgery encounters. Up to 41 International Classification of Diseases, 9th and 10th Revisions (ICD-9 and ICD-10) diagnoses and up to 41 ICD-9 and ICD-10 procedure codes are included. Billing data include medications, radiologic imaging studies, laboratory tests, and supplies charged to each patient. All data are deidentified at the time of data submission, and data are subjected to several reliability and validity checks before being included in the database.

The Children's Healthcare of Atlanta institutional review board and the Children's Hospital Association Board determined this study to be exempt from review because all patient-related data were deidentified before review and analysis.

\section{Study Population}

All inpatient and observational encounters of children 18 years of age or less, from January 1, 2008, to December 31, 2018, were collected from 31 hospitals that continuously contributed data to PHIS during the study period. One hospital was excluded for having chlorpromazine administered during a large proportion ( $10 \%)$ of PICU admissions, compared with less than $2 \%$ for all other hospitals, suggesting a potential for data errors. Children admitted to PICUs (and PCICUs, where applicable) with one of the selected antipsychotics were included in the final analysis. Antipsychotics identified for analysis included quetiapine, risperidone, ziprasidone, aripiprazole, olanzapine, chlorpromazine, and haloperidol.

Using all encounters in PHIS before the PICU or PCICU encounter, we excluded children with a concurrent ICD-9 or ICD-10 diagnosis code for a psychiatric condition, including schizotypal disorder, schizophrenia, schizoaffective disorder, delusional disorders, brief psychotic disorders, shared psychotic disorders, unspecified psychosis, mania, bipolar disorders, and major depressive disorders (-Supplementary Table S1; available in the online version).

Information about the PICU and hospital course collected included duration of mechanical ventilation, PICU length of stay, hospital length of stay, and hospital mortality. Demographics include age, sex, race and/or ethnicity (non-Hispanic white, non-Hispanic African American, Hispanic, or other), primary insurance payer (government, commercial or selfpay, or unknown), and admission diagnosis. Complex chronic conditions were defined by ICD-9 and ICD-10 codes and derived from hospitalization patterns of children with costly illnesses and congenital defects. ${ }^{21}$ Chronic conditions on ICU admission were subcategorized as $0,1,2$, or more than 3 conditions. The chronic conditions included any of the following: cardiovascular, gastrointestinal, hematologic/immunologic, malignancy, metabolic, neurologic/neuromuscular, congenital, renal/urologic, and respiratory.

\section{Statistical Analysis}

Antipsychotic medications were grouped into two categories: typical (haloperidol and chlorpromazine) and atypical (quetiapine, risperidone, ziprasidone, olanzapine, and aripiprazole). Descriptive statistics were calculated for all variables of interest. Categorical variables were summarized as counts or percentages. Continuous variables were summarized as medians with interquartile ranges (IQRs). To explore how patient demographics or antipsychotic use changed over time, we treated the discharge year as an ordinal variable. Differences between admissions with and without antipsychotic use were conducted using cluster-adjusted linear and logistic regression models. The type-3 fixed effect $p$-value for antipsychotic use as the main predictor was reported from these models. Standardized differences (effect sizes) were used to assess differences between admissions with and without antipsychotic use: effect sizes $<0.1$ were considered negligible, 0.1 to $<0.3$ was small, 0.3 to 0.75 was medium and $>0.75$ was large.

Usage trends were calculated using the Cochran-Armitage trend test with a one-sided $p$-value for testing increasing 
or decreasing trends over discharge years. Cluster-adjusted generalized regression models were used to test for trends over time, while adjusting for correlations of admissions within hospitals. Hospital-level variability of admissions using typical and atypical antipsychotics was tested by including a random intercept for hospital in generalized regression models. Standardized differences (effect sizes) were computed to assess differences between typical versus atypical antipsychotic admissions. A multivariable logistic regression model, also adjusting for admissions nested within hospitals, was used to estimate the adjusted odds of mortality. The main predictor was antipsychotic administration, and covariates that were controlled for included age of patient, sex, race, insurance type, chronic conditions on PICU admission, mechanical ventilation use, and admission type. $^{22}$ Analysis was conducted using statistical software (SAS version 9.4, SAS Institute Inc., Cary, North Carolina, United States). Statistical significance was assessed at the 0.05 level.

\section{Results}

\section{Population Characteristics}

During the study period, 759,659 PICU and PCICU admissions from 31 hospitals were evaluated. After exclusion of patients older than 18 years and those with a psychiatric diagnosis, we identified 16,465 admissions (2.2\%) for the antipsychotic subgroup analysis (-Fig. 1). Of the 16,465 admissions with antipsychotic use, 11,785 received an atypical antipsychotic, 3,321 received a typical antipsychotic, and 1,359 received

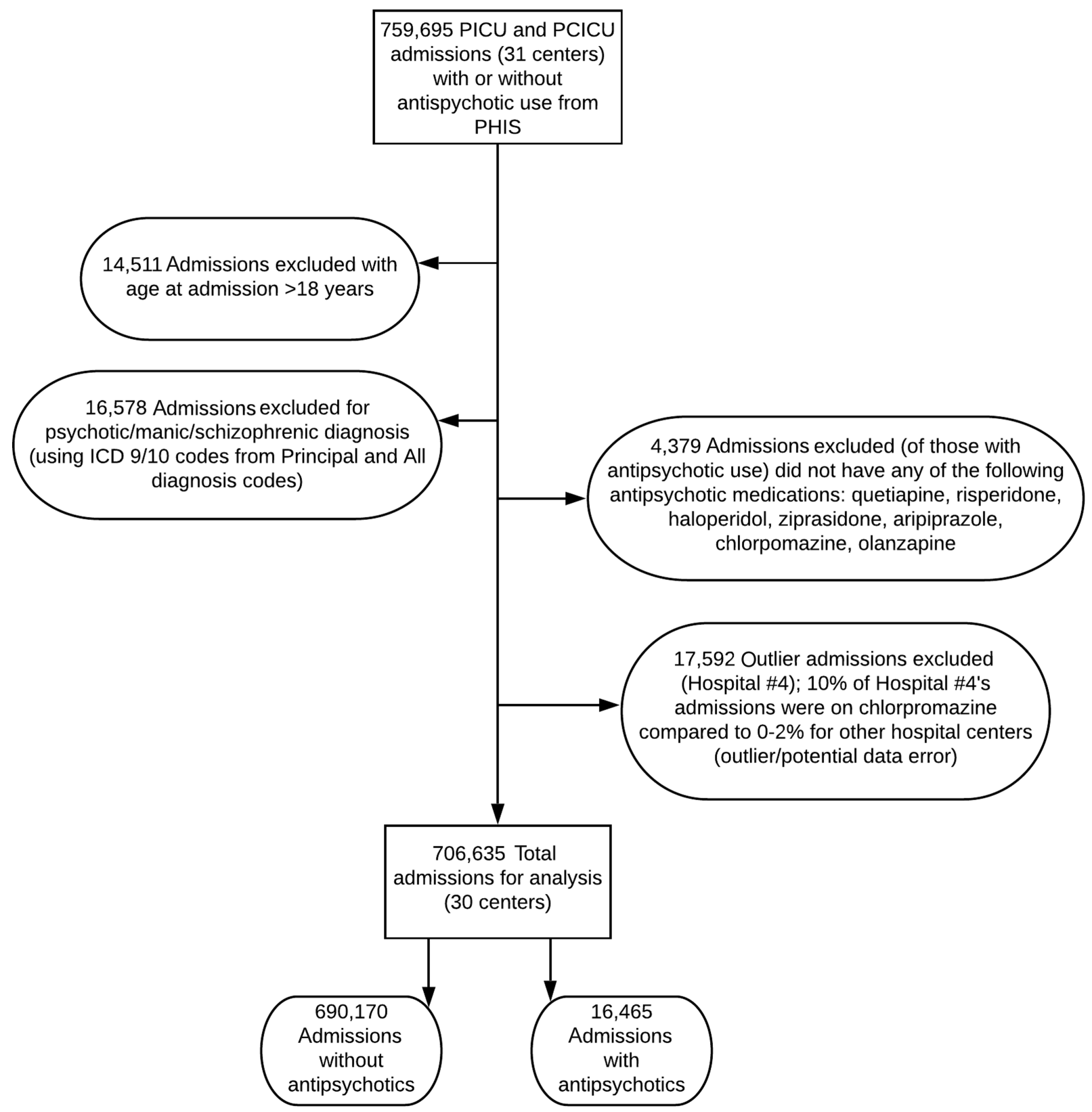

Fig. 1 Cohort flowchart. Details of cohort composition, starting with 759,695 admissions to pediatric intensive care units (PICUs) and pediatric cardiac intensive care units (PCICUs) in 31 centers. Abbreviation: ICD 9/10, International Classification of Diseases, 9th and 10th Revisions. 
both. The median age of patients who received antipsychotics was 11 years (IQR: $4-15$ years), with $25.5 \%$ aged 0 to 4 years, $37.9 \%$ female, and $59.0 \%$ having government insurance (-Table 1). The most common admission diagnoses were respiratory (13.7\%), trauma (12.2\%), neurologic (10.1\%), or other categories (37.8\%). Only $19.6 \%$ of admissions involved no chronic conditions, and $31.6 \%$ had more than two chronic conditions. The median ICU length of stay was 6 (IQR: 2-18) days, and overall hospital mortality was $5.7 \%$ (-Table 1). The median duration of antipsychotic administration was 4 days (IQR: 2-11 days), and patients received an antipsychotic for 44.4\% (IQR: 9.1-100\%) of their total ICU days.

Table 1 Clinical characteristics of pediatric intensive care unit (PICU) admissions in which antipsychotic used compared with no antipsychotic medication $^{\text {a }}$

\begin{tabular}{|c|c|c|c|c|c|}
\hline Characteristic & $\begin{array}{l}\text { All Admissions } \\
(\mathrm{N}=706,635)\end{array}$ & $\begin{array}{l}\text { Admissions With } \\
\text { Antipsychotics } \\
(n=16,465)\end{array}$ & $\begin{array}{l}\text { No Antipsychotics } \\
(n=690,170)\end{array}$ & $p$-Value & Effect Size $^{b}$ \\
\hline $\begin{array}{l}\text { Age at admission, months } \\
\text { median (IQR) }\end{array}$ & $42.6(8.5-129.8)$ & $136.3(57.8-184.2)$ & $41.1(8.2-126.9)$ & $<0.001$ & 0.750 \\
\hline \multicolumn{6}{|l|}{ Age groups, years (\%) } \\
\hline $0-4$ & $400,092(56.6)$ & $4,206(25.5)$ & $395,886(57.4)$ & $<0.001$ & 0.682 \\
\hline $5-9$ & $113,172(16.0)$ & $2,990(18.2)$ & $110,182(16.0)$ & $<0.001$ & 0.058 \\
\hline $10-14$ & $111,848(15.8)$ & $4,671(28.4)$ & $107,177(15.5)$ & $<0.001$ & 0.314 \\
\hline $15-18$ & $81,523(11.5)$ & 4,598 (27.9) & 76,925 (11.1) & $<0.001$ & 0.433 \\
\hline Female sex (\%) & $313,262(44.3)$ & 6,248 (37.9) & $307,014(44.5)$ & $<0.001$ & 0.133 \\
\hline \multicolumn{6}{|l|}{ Race/ethnicityc n(\%) } \\
\hline Non-Hispanic white & $356,503(51.8)$ & $9,618(59.6)$ & $346,885(51.6)$ & $<0.001$ & 0.161 \\
\hline Non-Hispanic Black & $138,427(20.1)$ & $2,626(16.3)$ & $135,801(20.2)$ & $<0.001$ & 0.102 \\
\hline Hispanic & $113,068(16.4)$ & 2,239 (13.9) & $110,829(16.5)$ & $<0.001$ & 0.073 \\
\hline Other & $80,601(11.7)$ & $1,664(10.3)$ & 78,937 (11.7) & $<0.001$ & 0.046 \\
\hline \multicolumn{6}{|l|}{ Insurance $n(\%)$} \\
\hline Government & $404,883(57.3)$ & $9,710(59.0)$ & $395,173(57.3)$ & $<0.001$ & 0.035 \\
\hline Private & $265,605(37.6)$ & $5,999(36.4)$ & $259,606(37.6)$ & $<0.001$ & 0.024 \\
\hline Other & $36,147(5.1)$ & $756(4.6)$ & $35,391(5.1)$ & $<0.001$ & 0.025 \\
\hline \multicolumn{6}{|l|}{ Admission diagnosis $\mathrm{n}(\%)$} \\
\hline Infectious/parasitic & $11,696(1.7)$ & $486(3.0)$ & $11,210(1.6)$ & $<0.001$ & 0.089 \\
\hline Hemato-oncologic & $30,405(4.3)$ & $1,005(6.1)$ & $29,400(4.3)$ & $<0.001$ & 0.083 \\
\hline Neurologic & $54,774(7.8)$ & $1,669(10.1)$ & $53,105(7.7)$ & $<0.001$ & 0.086 \\
\hline Cardiovascular & $25,315(3.6)$ & $756(4.6)$ & $24,559(3.6)$ & $<0.001$ & 0.052 \\
\hline Respiratory & $119,276(16.9)$ & $2,257(13.7)$ & $117,019(17.0)$ & $<0.001$ & 0.090 \\
\hline $\begin{array}{l}\text { Traumatic injury/external } \\
\text { causes of injury and } \\
\text { exposures }\end{array}$ & $61,411(8.7)$ & $2,014(12.2)$ & $59,397(8.6)$ & $<0.001$ & 0.119 \\
\hline Congenital malformations & $112,828(16.0)$ & $1,594(9.7)$ & $111,234(16.1)$ & $<0.001$ & 0.193 \\
\hline Ill-defined symptoms/signs & $147,937(20.9)$ & 3,605 (21.9) & $144,332(20.9)$ & $<0.001$ & 0.024 \\
\hline Other/unknown & $142,993(20.2)$ & $3,079(18.7)$ & $139,914(20.2)$ & $<0.001$ & 0.007 \\
\hline \multicolumn{6}{|l|}{$\begin{array}{l}\text { Chronic conditions on } \\
\text { admission }^{d} n(\%)\end{array}$} \\
\hline 0 & $243,940(34.5)$ & $3,221(19.6)$ & $240,719(34.9)$ & $<0.001$ & 0.349 \\
\hline 1 & $226,136(32.0)$ & $4.459(27.1)$ & $221,677(32.1)$ & $<0.001$ & 0.111 \\
\hline 2 & $122,957(17.4)$ & $3,588(21.8)$ & $119,369(17.3)$ & $<0.001$ & 0.114 \\
\hline$>2$ & $113,602(16.1)$ & $5,197(31.6)$ & $108,405(15.7)$ & $<0.001$ & 0.380 \\
\hline
\end{tabular}


Table 1 (Continued)

\begin{tabular}{|l|l|l|l|l|l|}
\hline Characteristic & $\begin{array}{l}\text { All Admissions } \\
(\mathbf{N}=706,635)\end{array}$ & $\begin{array}{l}\text { Admissions With } \\
\text { Antipsychotics } \\
(\mathbf{n}=16,465)\end{array}$ & $\begin{array}{l}\text { No Antipsychotics } \\
(\mathbf{n}=690,170)\end{array}$ & $p$-Value & Effect Size $^{\text {b }}$ \\
\hline $\begin{array}{l}\text { PICU LOS, days, }{ }^{\text {e }} \text { median } \\
\text { (IQR) }\end{array}$ & $3(2-6)$ & $6(2-18)$ & $3(2-6)$ & $<0.001$ & 0.354 \\
\hline $\begin{array}{l}\text { Hospital LOS, days, median } \\
\text { (IQR) }\end{array}$ & $6(4-12)$ & $14.0(6-37.0)$ & $6(4-11)$ & $<0.001$ & 0.481 \\
\hline $\begin{array}{l}\text { Mechanical ventilation } \\
\text { during PICU admission } \mathrm{n}(\%)\end{array}$ & $257,102(36.4)$ & $9,341(56.7)$ & $247,761(35.9)$ & $<0.001$ & 0.427 \\
\hline $\begin{array}{l}\text { Mechanical ventilation, days, } \\
\text { median (IQR) }\end{array}$ & $3(1-8)$ & $8(2-19)$ & $3(1-7.35)$ & $<0.001$ & 0.306 \\
\hline Hospital Mortality ${ }^{9} \mathrm{n}(\%)$ & $20,348(2.9)$ & $945(5.7)$ & $19,403(2.8)$ & $<0.001$ & 0.145 \\
\hline
\end{tabular}

aData are presented as number (percent) unless indicated by median (25th-75th percentiles). Some percentages do not total to 100 because of rounding.

bEffect size: standardized mean difference between the 2 groups. To compare each level, group variables with $>2$ levels were dummy coded at each level. Cohen D suggested that effect size indexes of $0.20,0.50$, and 0.80 represent small, medium, and large effect sizes.

'Missing race: $\mathrm{n}=18,036$ missing for all-admissions group.

${ }^{d}$ Chronic conditions include any of the following: cardiovascular, gastrointestinal, hematologic/immunologic, malignancy, metabolic, neurologic/neuromuscular, congenital, renal/urologic, and respiratory conditions.

${ }^{e}$ Missing ICU length of stay (LOS): $n=8,714$ missing for all-admissions group.

fDenominator is out of those with mechanical ventilation: ( $n=69,995$ missing days on mechanical ventilation if ventilated).

${ }^{9}$ Missing discharge status: $\mathrm{n}=13,647$ missing for all-admissions group.

\section{Comparison to General Pediatric Intensive Care Unit Population}

Use of antipsychotics during PICU admission, compared with no antipsychotics, was associated with older age (11 vs. 3 years; effect size $=0.745$ ), at least one comorbid condition (81vs. 65\%; effect size =0.39), longer PICU ( 6 vs. 3 days; effect size $=0.35$ ), and hospital length of stay ( 14 vs. 6 days; effect size $=0.48$ ). Use of antipsychotics during the PICU stay was also associated with mechanical ventilation support (57 vs. $36 \%$; effect size $=0.43$ ), with a median mechanical ventilation duration of 8 days (vs. 3 days in the no-antipsychotic group; effect size $=0.30$ ). In addition, the mortality of PICU admissions with antipsychotic administration was $5.7 \%$ compared with $2.8 \%$ overall for PICU admissions without antipsychotics (effect size $=0.145$ ). In a multivariable logistic regression model, adjusted for age, sex, race, insurance, chronic conditions, need for mechanical ventilation, and admission diagnosis group, odds ratio (OR) for mortality in PICU patients who received antipsychotics was 1.09 (95\% confidence interval [CI]: 1.0-1.18) compared with those who did not (-Table 2).

\section{Trends in Antipsychotic Use}

As shown in - Fig. 2, over the study period, use of quetiapine and olanzapine significantly increased $(p<0.001)$, whereas use of haloperidol, ziprasidone, and aripiprazole all decreased $(p<0.001)$. Use of risperidone $(p=0.363)$ and chlorpromazine $(p=0.106)$ did not change significantly over the study period when cluster-adjusted by hospital. When grouped as typical versus atypical antipsychotic medications, atypical antipsychotic use increased over the timeframe, and typical antipsychotic administration de- creased $(p<0.001)$. When cluster-adjusted by hospitals, both a positive trend in atypical antipsychotics and a negative trend in typical antipsychotics remained $(p<0.001)$.

\section{Variability in Antipsychotic Use across Hospitals}

-Fig. 3 shows the variability in antipsychotic use across the PICUs of the 30 PHIS hospitals included in the study. The PHIS institutions show a wide variability in use of atypical or typical antipsychotics as a percentage of total PICU admissions. For the 30 hospitals, the median proportion of ICU admissions during which a typical antipsychotic was prescribed was $0.45 \%$ (IQR: $0.39-0.74 \%$ ). Atypical antipsychotics were administered in a median of $1.68 \%$ of admissions (IQR: $1.38-2.11 \%$ ) across the 30 hospitals. Use of atypical agents occurred in less than $1 \%$ of admissions at two sites ( 8 and 13) and more than 3\% of admissions at sites 16 and 21 (-Fig. $\mathbf{3}$ ). Although most sites used typical agents in less than $1 \%$ of ICU admissions, a couple of sites ( 8 and 18) used them in more than $2 \%$ of admissions (-Fig. $\mathbf{3}$ ).

\section{Discussion}

Increased awareness of ICU delirium has consequently led to questions about delirium management, and the risks and benefits of pharmacologic therapy. ${ }^{8-17}$ Results of a recent randomized, double-blind, placebo-controlled trial of haloperidol and ziprasidone in adult ICU patients showed no difference in the duration of delirium. ${ }^{18}$ A clinical pathway published by Silver et $\mathrm{al}^{8}$ suggested a multidisciplinary approach to the prevention and management of delirium in children, and that antipsychotic drugs be given 
Antipsychotic Drug Prescription in U.S. PICUs Madden et al.

Table 2 Logistic regression models for odds of mortality with antipsychotic use versus no antipsychotic use

\begin{tabular}{|c|c|c|c|}
\hline \multicolumn{2}{|l|}{ Covariate } & \multirow{2}{*}{$\frac{\mathrm{OR}(95 \% \mathrm{Cl})}{1.09(1.02-1.18)}$} & \multirow{2}{*}{$\begin{array}{l}p \text {-Value } \\
0.017\end{array}$} \\
\hline Antipsychotic use & Yes vs. no & & \\
\hline \multirow[t]{4}{*}{ Age (y) } & $0-4$ & $1.01(0.96-1.06)$ & $<0.001$ \\
\hline & $5-9$ & $0.78(0.73-0.83)$ & $<0.001$ \\
\hline & $10-14$ & $0.89(0.83-0.94)$ & $<0.001$ \\
\hline & $15-18$ & Reference & \\
\hline Gender & Female vs. male & $1.05(1.02-1.08)$ & $<0.001$ \\
\hline \multirow[t]{4}{*}{ Race/ethnicity } & White & Reference & \\
\hline & Black & $1.09(1.05-1.14)$ & $<0.001$ \\
\hline & Hispanic & $1.1(1.05-1.15)$ & $<0.001$ \\
\hline & Other & $1.39(1.32-1.46)$ & $<0.001$ \\
\hline \multirow[t]{3}{*}{ Payer } & Private & Reference & \\
\hline & Government & $1.06(1.03-1.10)$ & $<0.001$ \\
\hline & Other & $1.71(1.6-1.8)$ & $<0.001$ \\
\hline \multirow[t]{4}{*}{ No. of chronic conditions } & 0 & Reference & \\
\hline & 1 & $2.53(2.38-2.69)$ & $<0.001$ \\
\hline & 2 & $4.43(4.17-4.7)$ & $<.001$ \\
\hline & $>2$ & $5.21(4.92-5.52)$ & $<0.001$ \\
\hline Mechanical ventilation & Yes vs. no & $19.6(18.6-20.7)$ & $<0.001$ \\
\hline \multirow[t]{10}{*}{ Admission category } & Unknown & Reference & \\
\hline & Infection & $2.09(1.85-2.37)$ & $<0.001$ \\
\hline & Heme-Onc & $2.1(1.92-2.4)$ & $<0.001$ \\
\hline & Cardiovascular & $0.87(0.78-0.98)$ & $<0.001$ \\
\hline & Neurologic & $3.58(3.33-3.98)$ & $<0.001$ \\
\hline & Respiratory & $0.76(0.69-0.85)$ & $<0.001$ \\
\hline & Trauma/exposures & $2.39(2.16-2.66)$ & $<0.001$ \\
\hline & Congenital & $0.57(0.52-0.64)$ & $<0.001$ \\
\hline & Ill-defined symptoms & $0.93(0.84-1.03)$ & $<0.001$ \\
\hline & Other & $1.21(1.09-1.34)$ & $<0.001$ \\
\hline
\end{tabular}

Abbreviations: $\mathrm{Cl}$, confidence interval; OR, odds ratio.

consideration, especially when the child is agitated. Here, we report continued use of antipsychotics in pediatric critical care, and an increasing trend in the atypical agents, like quetiapine and olanzapine, with a decrease in the use of typical agents like haloperidol.

The increase in prescription of atypical antipsychotics in PICU patients is in the context of a lack of FDA approval for use in children under 5 years or for management of delirium in children or adults. Several reports have demonstrated the safe administration of these agents in young children; however, their efficacy in altering clinical outcomes remains in question. ${ }^{12-17,23}$ The safety profile of atypical antipsychotics is improved compared with typical agents, with fewer extrapyramidal symptoms, lower incidence of neuroleptic malignant syndrome, less hepatic dysfunction, and fewer drug interactions. ${ }^{15,24,25}$ However, adverse effects, such as weight gain, insulin resistance, and hyperlipidemia, do occur. ${ }^{24,26-29}$ These develop over a longer period than an usual
ICU stay; however, agents started in the ICU may continue after transfer or discharge. Additionally, the impact of these agents on longer term brain development when used in younger children is not completely understood

It was not surprising that one-fourth of antipsychotics were prescribed for children under 4 years of age, because this group is at the highest risk of delirium according to several PICU series. ${ }^{2,6,30}$ Likewise, patients receiving mechanical ventilation and with admission diagnoses of trauma or respiratory or neurologic illness were more commonly prescribed antipsychotics in our study. This is similar to the results of an international point prevalence study on delirium in which children receiving antiepileptic medications and those receiving sedation and analgesia were at highest risk of delirium. ${ }^{2}$ We also found that a high proportion of admissions with use of antipsychotic agents involved in multiple chronic conditions compared with the general PICU population. This is of interest because Dechnik and 


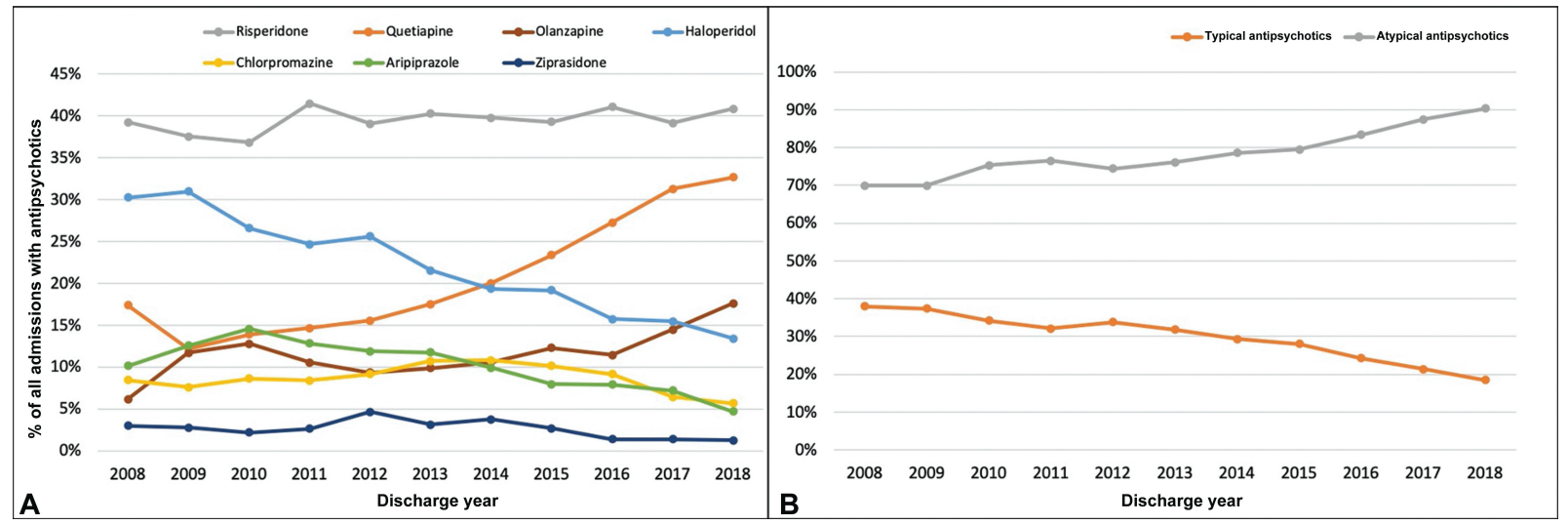

Fig. 2 Trends of antipsychotic use, 2008-2018. Pediatric intensive care unit (PICU) and pediatric cardiac intensive care unit (PCICU) admissions for 16,465 children using Cochran-Armitage trend test. (A) Specific antipsychotics. Positive trends for quetiapine, risperidone, and olanzapine. (B) Typical versus atypical antipsychotics. Positive trend for atypical antipsychotics $(p<0.001)$.

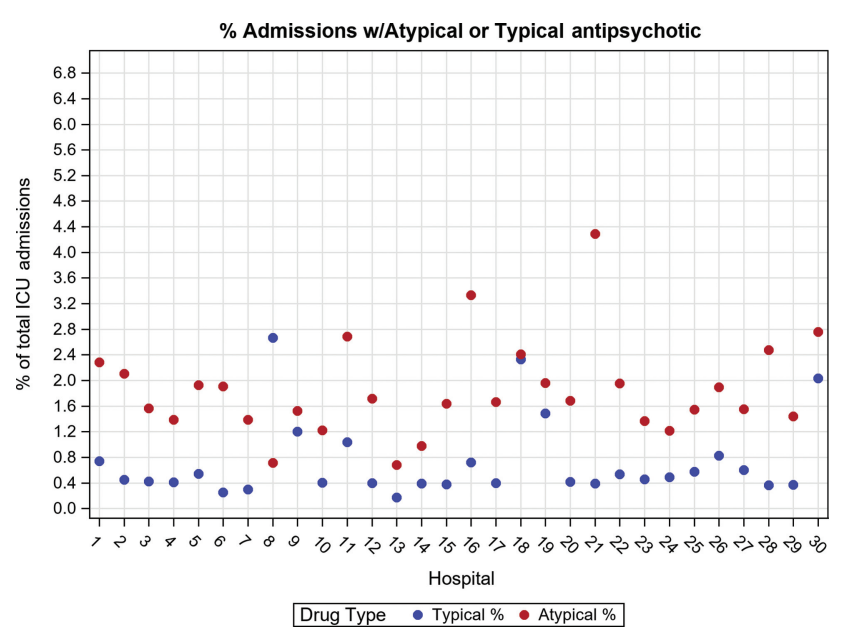

Fig. 3 Proportion of pediatric intensive care unit (PICU) admissions with antipsychotic use by hospital. Each of the 30 pediatric health information system database centers is designated by a hospital number.

Traube $^{31}$ reported that patients with preexisting chronic conditions, poor nutritional status, or cognitive or neurologic disabilities are at higher risk of delirium.

While antipsychotic prescription was associated with increased comorbidity, ventilation, and longer PICU length of stay, controlling for these features in the multivariable model demonstrated a persistent relationship between antipsychotic administration and mortality. Data limitation does not allow us to imply a cause and effect relationship to the antipsychotic agent. It is likely a combined effect of increased comorbidities, complexity of PICU admission, and mechanical ventilation, all associated with increased delirium leading to antipsychotic use. The high rate of chronic comorbidities in the PICU population reported here also likely reflects a degree of polypharmacy in these patients, and a high risk of drug interaction. We believe this relationship deserves future study. Although there are limitations of using mortality as the outcome measure for pediatric critical illness, limitations of the data available in the PHIS database meant we were unable to fully explore other potential outcomes in the cohort.
Interestingly, patients who received antipsychotic agents did so for almost $45 \%$ of their PICU length of stay. The duration of use for quetiapine in this study was 6 days (IQR: 3-15 days), much lower than reported in a prior study in which median quetiapine use was 12 days (IQR: 4.5-22 days) in critically ill children. ${ }^{12}$ We also report a variability in the use of antipsychotics (typical vs. atypical) across the PHIS hospitals, with use of atypical agents ranging from less than $1 \%$ to more than $3 \%$ of ICU admissions, and typical agents in less than $1 \%$ to more than $2 \%$ of admissions. This variability likely reflects varying case mix across hospitals, as well as comfort in the diagnosis of delirium, pharmacologic treatment of delirium, atypical versus typical agents, and use of nonpharmacologic therapies.

\section{Limitations}

There are several limitations to using the PHIS database which have previously been described by other studies. ${ }^{32,33}$ It is an administrative database, and although it provides more therapeutic and diagnostic data per patient than most administrative datasets, no physiologic data are available. Because the children's hospitals participating in the PHIS are tertiary and quaternary care facilities in major metropolitan cities in the United States, the data are likely not generalizable to community hospitals or hospitals that do not offer specialty pediatric services. We were also not able to look at the continuation of antipsychotics after ICU discharge or transfer or to compare the trends in antipsychotic use in pediatric critical care to wider inpatient and outpatient usage of these medications. Furthermore, the study is subject to the limitations of all observational analyses including selection bias, residual confounding, and measurement error. There may be inherent differences in the coding practices among PHIS-participating hospitals that contribute to selection bias which may be minimized by the use of previously described and, in some cases, validated ICD-9 and ICD-10 codes. Because these codes notoriously insufficient to measure true incidence of diagnoses in hospitalized patients, we were not able to accurately measure rates of delirium diagnosis and the relationship of that timing to antipsychotic 
administration, so we chose not to interrogate the assignment of delirium diagnosis codes in this cohort. We are not able to definitively report that these antipsychotics were all administered to treat delirium, although that is our experience of their use as PICU clinicians.

\section{Conclusion}

Over the past 10 years, use of atypical antipsychotics, including quetiapine and olanzapine, have increased, whereas haloperidol use has decreased. This may reflect increasing comfort with atypical agents which are not FDA approved in the treatment of delirium. Chronic comorbidities and mechanical ventilation support were associated with greater odds of antipsychotic medication use in the PICU, and antipsychotic use was correlated with higher mortality when controlling for other available clinical factors. Because of limitations to the database, we are not able to draw a cause and effect relationship between antipsychotic use or delirium and mortality; however, further study is needed to systematically assess this relationship in critically ill patients.

\section{Authors' Contributions}

K.M., R.C.T., J.F., C.M., and P.K. participated in the concept and design, analysis and interpretation of data, and drafting or revising of the manuscript. M.W. and M.H. participated in interpretation of data and revising of the manuscript. All authors have approved the final manuscript as submitted and agree to be accountable for all aspects of the work.

\section{Funding}

None.

\section{Conflict of Interest \\ None delcared.}

\section{Acknowledgments}

The authors wish to acknowledge the contributions of Dennis Sullivan, MS, formerly of Children's Healthcare of Atlanta, in the design of this study, initial data collection and analysis, and PHIS expertise in general.

\section{References}

1 Alvarez RV, Palmer C, Czaja AS, et al. Delirium is a common and early finding in patients in the pediatric cardiac intensive care unit. J Pediatr 2018;195:206-212

2 Traube C, Silver G, Reeder RW, et al. Delirium in critically ill children: an international point prevalence study. Crit Care Med 2017;45(04):584-590

3 Bryant KJ. Pediatric delirium in the cardiac intensive care unit: identification and intervention. Crit Care Nurse 2018;38(04): e1-e7

4 Patel AK, Biagas KV, Clarke EC, et al. Delirium in children after cardiac bypass surgery. Pediatr Crit Care Med 2017;18(02): 165-171

5 Meyburg J, Dill ML, Traube C, Silver G, von Haken R. Patterns of postoperative delirium in children. Pediatr Crit Care Med 2017;18 (02):128-133
6 Traube C, Silver G, Gerber LM, et al. Delirium and mortality in critically ill children: epidemiology and outcomes of pediatric delirium. Crit Care Med 2017;45(05):891-898

7 Meyburg J, Dill ML, von Haken R, et al. Risk factors for the development of postoperative delirium in pediatric intensive care patients. Pediatr Crit Care Med 2018;19(10):e514-e521

8 Silver GH, Kearney JA, Bora S, et al; PATHWAYS FOR CLINICAL CARE WORKGROUP. A clinical pathway to standardize care of children with delirium in pediatric inpatient settings. Hosp Pediatr 2019;9(11):909-916

9 Luetz A, Grunow JJ, Mörgeli R, et al. Innovative ICU solutions to prevent and reduce delirium and post-intensive care unit syndrome. Semin Respir Crit Care Med 2019;40(05):673-686

10 Simone S, Edwards S, Lardieri A, et al. Implementation of an ICU bundle: an interprofessional quality improvement project to enhance delirium management and monitor delirium prevalence in a single PICU. Pediatr Crit Care Med 2017;18(06): 531-540

11 Kishk OA, Simone S, Lardieri AB, Graciano AL, Tumulty J, Edwards S. Antipsychotic treatment of delirium in critically ill children: a retrospective matched cohort study. J Pediatr Pharmacol Ther 2019;24(03):204-213

12 Joyce C, Witcher R, Herrup E, et al. Evaluation of the safety of quetiapine in treating delirium in critically ill children: a retrospective review. J Child Adolesc Psychopharmacol 2015;25(09): 666-670

13 Traube C, Witcher R, Mendez-Rico E, Silver G. Quetiapine as treatment for delirium in critically ill children: a case series. J Pediatr Intensive Care 2013;2(03):121-126

14 Sassano-Higgins S, Freudenberg N, Jacobson J, Turkel S. Olanzapine reduces delirium symptoms in the critically ill pediatric patient. J Pediatr Intensive Care 2013;2(02):49-54

15 Slooff VD, Spaans E, van Puijenbroek E, et al. Adverse events of haloperidol for the treatment of delirium in critically ill children. Intensive Care Med 2014;40(10):1602-1603

16 Slooff VD, van den Dungen DK, van Beusekom BS, et al. Monitoring haloperidol plasma concentration and associated adverse events in critically ill children with delirium: first results of a clinical protocol aimed to monitor efficacy and safety. Pediatr Crit Care Med 2018;19(02):e112-e119

17 Cronin MT, Di Gennaro JL, Watson RS, Dervan LA. Haloperidol and quetiapine for the treatment of ICU-associated delirium in a tertiary pediatric ICU: a propensity score-matched cohort study. Paediatr Drugs 2021;23(02):159-169

18 Girard TD, Exline MC, Carson SS, et al; MIND-USA Investigators. Haloperidol and ziprasidone for treatment of delirium in critical illness. N Engl J Med 2018;379(26):2506-2516

19 Daniels LM, Nelson SB, Frank RD, Park JG. Pharmacologic treatment of intensive care unit delirium and the impact on duration of delirium, length of intensive care unit stay, length of hospitalization, and 28-day mortality. Mayo Clin Proc 2018;93(12): 1739-1748

20 Devlin JW, Roberts RJ, Fong JJ, et al. Efficacy and safety of quetiapine in critically ill patients with delirium: a prospective, multicenter, randomized, double-blind, placebo-controlled pilot study. Crit Care Med 2010;38(02):419-427

21 Feudtner C, Feinstein JA, Zhong W, Hall M, Dai D. Pediatric complex chronic conditions classification system version 2: updated for ICD-10 and complex medical technology dependence and transplantation. BMC Pediatr 2014;14:199

22 Maldonado JR. Acute brain failure: pathophysiology, diagnosis, management, and sequelae of delirium. Crit Care Clin 2017;33 (03):461-519

23 Capino AC, Thomas AN, Baylor S, Hughes KM, Miller JL, Johnson PN. Antipsychotic use in the prevention and treatment of intensive care unit delirium in pediatric patients. J Pediatr Pharmacol Ther 2020;25(02):81-95 
24 Ames D, Carr-Lopez SM, Gutierrez MA, et al. Detecting and managing adverse effects of antipsychotic medications: current state of play. Psychiatr Clin North Am 2016;39(02):275-311

25 Kudo S, Ishizaki T. Pharmacokinetics of haloperidol: an update. Clin Pharmacokinet 1999;37(06):435-456

26 Newcomer JW. Second-generation (atypical) antipsychotics and metabolic effects: a comprehensive literature review. CNS Drugs 2005;19(Suppl 1):1-93

27 Nicol GE, Yingling MD, Flavin KS, et al. Metabolic effects of antipsychotics on adiposity and insulin sensitivity in youths: a randomized clinical trial. JAMA Psychiatry 2018;75(08):788-796

28 Kowalchuk C, Castellani LN, Chintoh A, Remington G, Giacca A, Hahn MK. Antipsychotics and glucose metabolism: how brain and body collide. Am J Physiol Endocrinol Metab 2019;316(01):E1-E15
29 Holt RIG. Association between antipsychotic medication use and diabetes. Curr Diab Rep 2019;19(10):96

30 Smith HA, Gangopadhyay M, Goben CM, et al. The preschool confusion assessment method for the ICU: valid and reliable delirium monitoring for critically ill infants and children. Crit Care Med 2015

31 Dechnik A, Traube C. Delirium in hospitalised children. Lancet Child Adolesc Health 2020;4(04):312-321

32 Geanacopoulos AT, Porter JJ, Monuteaux MC, Lipsett SC, Neuman MI. Trends in chest radiographs for pneumonia in emergency departments. Pediatrics 2020;145(03):145

33 Synhorst DC, Johnson MB, Bettenhausen JL, et al. Room costs for common pediatric hospitalizations and cost-reducing quality initiatives. Pediatrics 2020;145(06):145 